\title{
SUPERCONDUCTING PROTOTYPE CAVITIES FOR THE SPALLATION NEUTRON SOURCE (SNS) PROJECT *
}

\author{
G. Ciovati, P. Kneisel ${ }^{\dagger}$, J. Brawley, R. Bundy, I. Campisi, K. Davis, K. Macha, D. Machie, J. \\ Mammosser, S. Morgan, R. Sundelin, L. Turlington, K. Wilson, Jefferson Lab; M. Doleans, S.H. \\ Kim, D. Mangra, ORNL-SNS; D. Barni, C. Pagani, P. Pierini, INFN Milano; K. Matsumoto, R. \\ Mitchell, D. Schrage, LANL; R. Parodi, INFN Genova; J. Sekutowicz, DESY; P. Ylae-Oijala, \\ Univ. Helsinki
}

\begin{abstract}
The Spallation Neutron Source project includes a superconducting linac section in the energy range from $186 \mathrm{MeV}$ to $1000 \mathrm{MeV}$. For this energy range two types of cavities are needed with geometrical $\beta$ values of $\beta=0.61$ and $\beta=0.81$. An aggressive cavity prototyping program is being pursued at Jefferson Lab, which calls for fabricating and testing four $\beta=0.61$ cavities and two $\beta=0.81$ cavities. Both types consist of six cells made from high purity niobium and feature one HOM coupler of the TESLA type on each beam pipe and a port for a high power coaxial input coupler. Three of the four $\beta=0.61$ cavities will be used for a cryomodule test at the end of 2001. Two cavities of each type have been fabricated and the first tests on both cavities exceeded the design values for gradient and $Q$ value: $E_{\text {acc }}=10.1 \mathrm{MV} / \mathrm{m}$ and $Q=5 \times 10^{9}$ at $2.1 \mathrm{~K}$ for the $\beta=0.61$ and $E_{\text {acc }}=12.5$ $\mathrm{MV} / \mathrm{m}$ and $Q=5 \times 10^{9}$ at $2.1 \mathrm{~K}$ for the $\beta=0.81$.
\end{abstract}

\section{ELECTROMAGNETIC DESIGN}

The designs for the two types of SNS cavities were dominated by the desire to keep the electric peak surface field at or below $27.5 \mathrm{MV} / \mathrm{m}$ and the magnetic peak surface field below $60 \mathrm{mT}$. At the same time the Lorentz force detuning coefficient $\left(K_{\mathrm{L}}\right)$ should not exceed a value of $-3 \mathrm{~Hz} /(\mathrm{MV} / \mathrm{m})^{2}$. By choosing a cell-to-cell coupling of $1.5 \%$ for these six-cell cavities a reasonably small ratio of peak surface fields and accelerating fields could be realized [1], if four different half cell shapes were used. The fundamental power coupler (FPC) requirement for a $Q_{\text {ext }}$ value of about $10^{6}$ implied larger iris and equator diameters for the end half cells at the FPC side (see figure 1) compared to the center cells. Simulation calculations done with the center cell geometries and the full cavities showed that multipacting was unlikely. The specifications for the Lorentz coefficient require welded stiffening rings between the cell irises at a radius of $80 \mathrm{~mm}$. The SNS cavities have Nb55Ti flanges throughout (DESY-type), sealed with $\mathrm{AlMg}_{3}$ gaskets: one at each tapered beam pipe end, two ports (one for each end) for the HOM couplers, rotated 115 degrees with respect to each other, a port for the field probe and one for the FPC. The coaxial FPC is the KEK design scaled to $805 \mathrm{MHz}$ [2].

\footnotetext{
* Work supported by the U.S. DOE Contract No DE-AC05-00-OR22725 †kneisel@jlab.org
}

Table 1: SNS cavities' electromagnetic parameters.

\begin{tabular}{|l|c|c|}
\hline \multicolumn{1}{|c|}{ Cavity $\beta$} & $\mathbf{0 . 6 1}$ & $\mathbf{0 . 8 1}$ \\
\hline Frequency $[\mathrm{MHz}]$ & 805.000 & 805.000 \\
\hline$E_{\text {peak }} / E_{\text {acc }}$ & 2.71 & 2.19 \\
\hline$B_{\text {peak }} / E_{\text {acc }}[\mathrm{mT} /(\mathrm{MV} / \mathrm{m})]$ & 5.72 & 4.72 \\
\hline$R / Q[\Omega]$ & 279 & 483 \\
\hline$G\left(=R_{\mathrm{s}} Q_{0}\right)[\Omega]$ & 179 & 260 \\
\hline Cell-to-cell $k[\%]$ & 1.53 & 1.52 \\
\hline$K_{\mathrm{L}}\left[\mathrm{Hz} /(\mathrm{MV} / \mathrm{m})^{2}\right]$ & -2.07 & -0.43 \\
\hline
\end{tabular}

\section{FABRICATION}

The SNS cavity cells are fabricated from RRR $>250$ niobium. The dumbbells exhibited significant mechanical deformations after the attachment of the stiffening rings. This required re-adjustments of mechanical dimensions and frequencies of the dumbbells in a way similar to that described in [3]. The dumbbell had to be stretched and trimmed at the equator to reach both the right length and frequency. Once the dumbbells had been corrected, the electron beam welding of the cavity assembly started from the two end groups by adding dumbbells; the last weld was the equator weld of the two half sub-assemblies.

Tuning to a "flat" field profile followed a $20 \mu \mathrm{m}$ external chemistry. Internal buffered chemical polishing (BCP) in JLab's closed chemistry system with $\sim 120 \mu \mathrm{m}$ material removal and subsequent high pressure ultrapure water rinsing for $2 \mathrm{~h}$ completed the final surface treatment. After drying for several hours the cavity was assembled in the clean room.

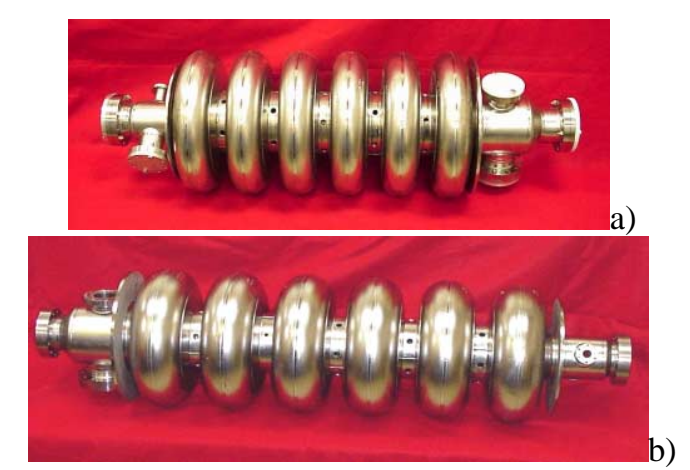

Figure 1: SNS $\beta=0.61$ (a) and $\beta=0.81$ (b) cavities. 


\section{VERTICAL TEST RESULTS}

The results of the vertical test for the six-cells $\beta=0.61$ cavity at $1.96 \mathrm{~K}$ are shown in figure 2 . The cavity had a $Q_{0}$ at low field of about $2.3 \times 10^{10}$ corresponding to a residual surface resistance of $2.1 \mathrm{n} \Omega$. No multipacting barrier has been found and, after about $20 \mathrm{~min}$ of $\mathrm{RF}$ processing, the cavity reached $E_{\text {acc }}=16 \mathrm{MV} / \mathrm{m}$ with a $Q$ of $1.4 \times 10^{10}$, exceeding comfortably the design goal for this cavity type.

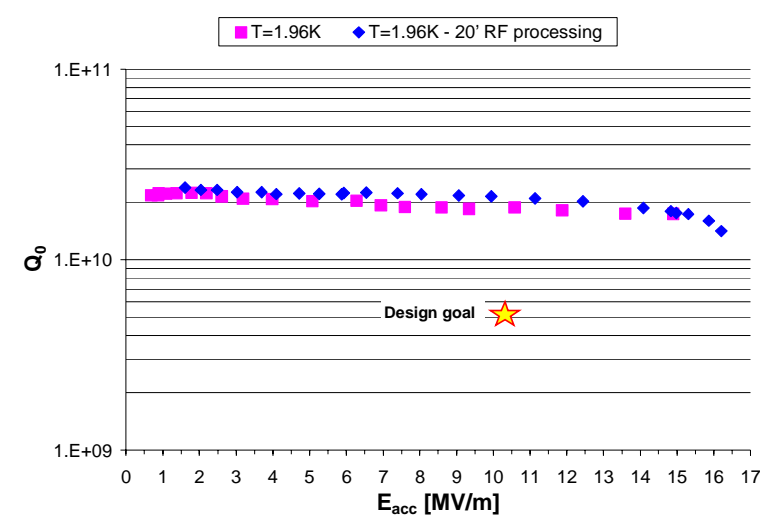

Figure 2: SNS $\beta=0.61$ vertical test results.

The results for the six-cells $\beta=0.81$ cavity at three different temperatures are shown in figure 3 . The $Q_{0}$ at low field was about $2.9 \times 10^{10}$. From the temperature dependence of the surface resistance a residual resistance of $7.9 \mathrm{n} \Omega$ was extracted. Also this cavity did not show any multipacting as predicted, but field emission started at about $E_{\text {acc }}=13 \mathrm{MV} / \mathrm{m}$. After about $20 \mathrm{~min}$ of $\mathrm{He}$ processing the cavity reached $E_{\text {acc }}=19 \mathrm{MV} / \mathrm{m}$ with a $\mathrm{Q}$ of $8.3 \times 10^{9}$.

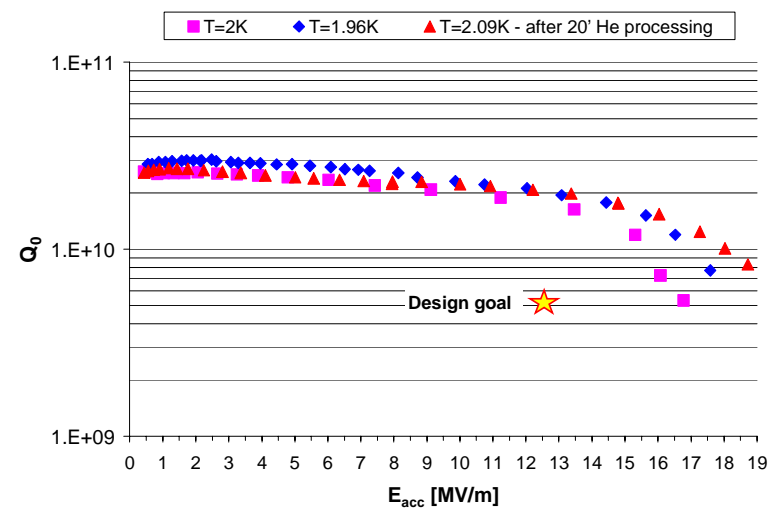

Figure 3: SNS $\beta=0.81$ vertical test results.

\section{MECHANICAL ANALYSIS}

\subsection{Lorentz force detuning}

During the cryogenic tests the cavities are supported in a Ti frame; measurements of the Lorentz force coefficient for both cavity types gave values of $-8.25 \mathrm{~Hz} /(\mathrm{MV} / \mathrm{m})^{2}$ for the $\beta=0.61$ cavity (the stiffening rings for this prototype cavity were located at $70 \mathrm{~mm}$ from the cavity axis) as shown in figure 4 and $-3.5 \mathrm{~Hz} /(\mathrm{MV} / \mathrm{m})^{2}$ for the high beta cavity. These results deviated significantly from the original finite element (FE) calculations, which used a Fixed end boundary condition.

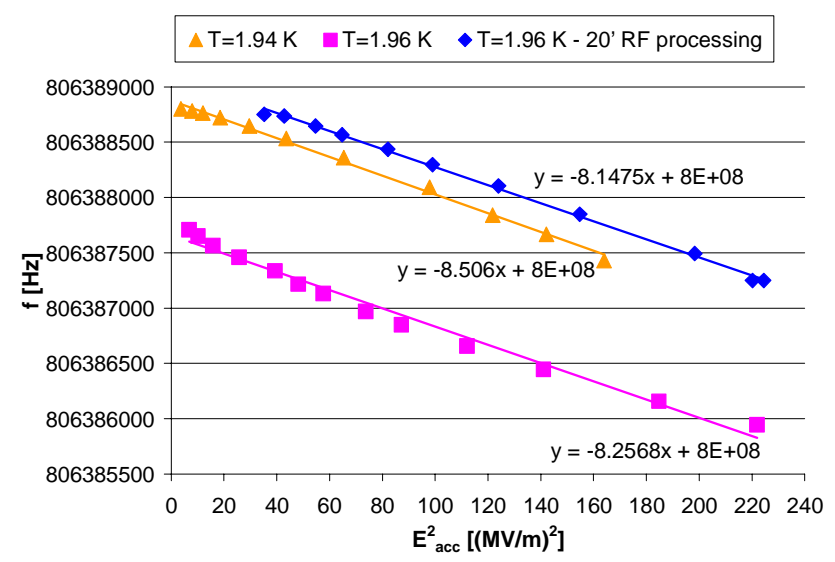

Figure 4: SNS $\beta=0.61$ frequency vs. $E_{\text {acc }}{ }^{2}$.

SUPERFISH was used to compute radiation pressures on the walls of the cavities and the resonant frequencies. With the finite element code ABAQUS the shape deformations due to the Lorentz pressure were calculated for an accurate model of the SNS cavities. The results from the calculations shows that the Lorentz force coefficient is strongly influenced by the boundary conditions the cavities are subjected to and changes by about one order of magnitude from fixed to free cavity end. Since the SNS cavities are supported in a titanium frame when they are tested in the vertical dewar, the spring constant of this fixture has been evaluated and used in the computations to reproduce the vertical test set-up. The values for the Lorentz force coefficient obtained in this configuration are shown in table 2 and are within $15 \%$ of the experimental ones.

Table 2: Lorentz coefficient $K_{\mathrm{L}}\left[\mathrm{Hz} /(\mathrm{MV} / \mathrm{m})^{2}\right]$ measured and computed with different boundary conditions.

\begin{tabular}{|c|c|c|c|c|}
\hline Cavity $\beta$ & \multicolumn{2}{|c|}{$\mathbf{0 . 6 1}$} & \multicolumn{2}{c|}{$\mathbf{0 . 8 1}$} \\
\hline & No stiff & $70 \mathrm{~mm}$ & No stiff & $80 \mathrm{~mm}$ \\
\hline Fixed end & -2.89 & -1.65 & -0.78 & -0.43 \\
\hline Ti frame & -7.85 & -7.0 & -3.62 & -3.5 \\
\hline Measured & & -8.25 & & -3.5 \\
\hline He vessel & -4.0 & -3.55 & -1.76 & -1.58 \\
\hline Free end & -31.1 & -27.0 & -12.2 & -10.1 \\
\hline
\end{tabular}

The stiffness of the He vessel and tuner has also been evaluated and used as boundary condition to calculate the Lorentz force coefficient. The results indicate that for the high beta cavity the $K_{\mathrm{L}}$ specifications will be met, whereas for the $\beta=0.61$ cavity additional stiffening of the helium vessel end dishes is necessary. 


\subsection{Natural Frequencies}

A natural frequency vibration test was performed on the prototype medium beta niobium cavity, supported with nylon straps at the ends of the beam tubes. A shaker was fixed onto the equator of one of the center cells (figure 5) and a tri-axial accelerometer was attached to an adjacent cell. An additional axial accelerometer was mounted to the FPC-end flange. (See figure 5.)

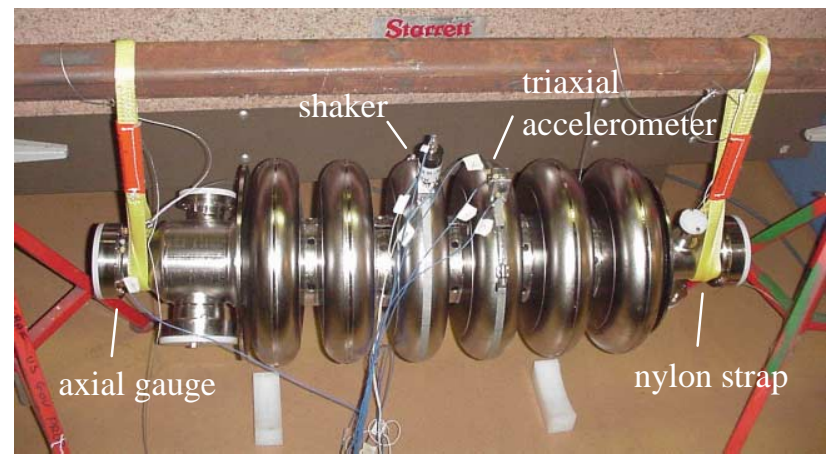

Figure 5: $\beta=0.61$ natural frequency vibration test set-up.

ABAQUS was used again to model the natural frequency spectrum of the cavity; the computed frequencies are in close agreement with the test results as listed in table 3. To help provide insight into the natural frequencies of the cavity within the cryomodule assembly, the ABAQUS cavity model will be extended to include cryomodule components. The same work is in progress for the high beta cavity.

Table 3: Comparison of test data and finite element analysis.

\begin{tabular}{|c|c|c|}
\hline & \multicolumn{2}{|c|}{ Natural frequencies [Hz] } \\
\hline Mode & Test data & FE Analysis \\
\hline 1 & 13 & 14 \\
\hline 2 & 31 & 26 \\
\hline 3 & 38 & 40 \\
\hline 4 & 53 & 48 \\
\hline 5 & 70 & 72 \\
\hline 6 & 82 & 83 \\
\hline 7 & 125 & 124 \\
\hline
\end{tabular}

\section{HIGH ORDER MODES ANALYSIS}

HOM calculations have been done with MAFIA and SUPERFISH for both types of cavities. Three TM monopole modes for each cavity have frequency close to a bunch harmonic and need adequate damping [4].

Table 4: Measured $Q_{\text {ext }}$ and frequencies for the dangerous modes on the $\beta=0.61$ cavity.

\begin{tabular}{|c|c|c|c|}
\hline Mode & $\begin{array}{c}\text { Freq. } \\
\text { [MHz] }\end{array}$ & $\begin{array}{c}\boldsymbol{Q}_{\text {ext }} \text { [ } \\
\text { FPC side }\end{array}$ & $\begin{array}{c}\boldsymbol{Q}_{\text {ext }} \text { @ } \\
\text { probe side }\end{array}$ \\
\hline $\mathrm{TM}_{021}-5 \pi / 6(\# 31)$ & 2818.79 & $2 \times 10^{5}$ & $4 \times 10^{4}$ \\
\hline $\mathrm{TM}_{021}-\pi(\# 32)$ & 2837.93 & $2.7 \times 10^{5}$ & $5 \times 10^{5}$ \\
\hline Beam pipe mode & 3221.30 & $2.5 \times 10^{3}$ & - \\
\hline
\end{tabular}

These modes have been identified on the copper models and have been damped with high order mode couplers of the DESY-type after optimization of the orientation as shown in table 4 for the medium $\beta$ cavity. Work on the high $\beta$ copper model is in progress.
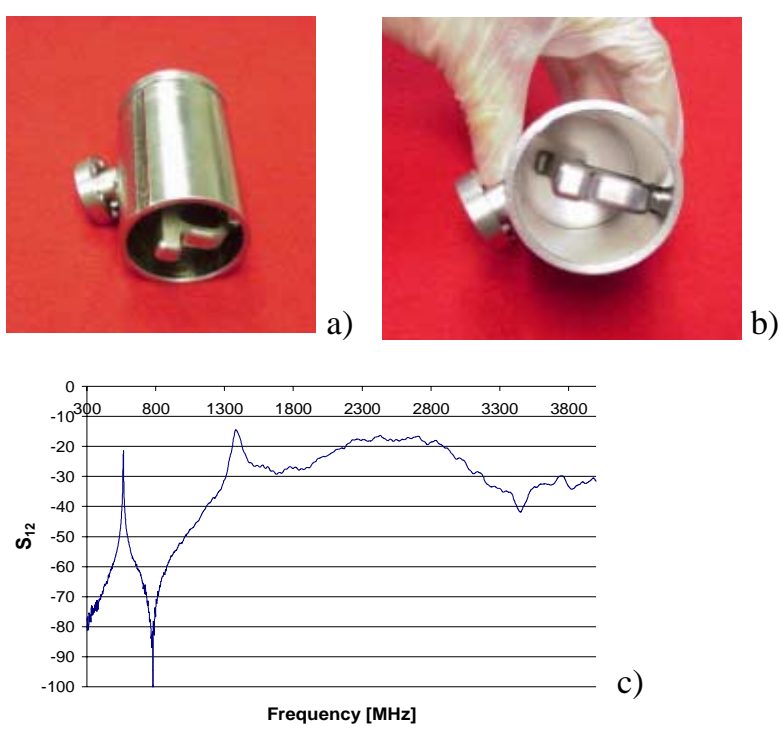

Figure 6: HOM filter (a), (b) and filter characteristic (c).

\section{SUMMARY}

It had been demonstrated that in both types of SNS cavities the design goals for $Q$-value and accelerating gradient can be achieved with some comfortable margin. Multipacting is no problem and the dangerous higher order modes can be damped appropriately. The mechanical behavior of the cavities has been analyzed; as a result, further stiffening of the helium vessel is necessary to achieve the specified values for the Lorentz force detuning. The three medium beta cavities needed for the prototype cryomodule are $80 \%$ complete at this time.

\section{REFERENCES}

[1] P. Pierini et al., "SC Cavity Design for the $700 \mathrm{MHz}$ TRASCO Linac", EPAC '00, Vienna, June 26-30, 2000.

[2] Y. Kang et al., "Electromagnetic Simulations and Properties of the Fundamental Power Couplers for the SNS Superconducting Cavities", these proceedings.

[3] G. Kreps, D. Proch and J. Sekutowicz, "Half Cell and Dumbbell Frequency Testing for the Correction of the TESLA Cavity Length", 9 ${ }^{\text {th }}$ RF Superconductivity Workshop, Santa Fe, Nov. 1-5, 1999, paper WEP031.

[4] S-H. Kim, R. Sundelin, "SNS HOM Damping Requirements via Bunch Tracking", these proceedings.

\section{ACKNOWLEDGEMENT}

We would like to thank all our colleagues who contributed to this work. 